Als Beisitzer wurden gewählt: Peter Duhr (Studierendeninitiative IGUWi, Oldenburg), Dipl. oec. Simone Maier (Hochschule St. Gallen/Schweiz), Dipl. Vw. Jan Nill (Institut für ökologische Wirtschaftsforschung, Berlin), Prof. Dr. Reinhard Pfriem (Universität Oldenburg), Prof. Dr. Stefan Schaltegger (Universität Lüneburg), Prof. Dr. Uwe Schneidewind (Universität Oldenburg), sowie Dipl. Biol. Dietrich Weber (Bonn).

Arbeitsschwerpunkte des neu gewählten Vorstandes liegen in der Verbesserung der Öffentlichkeitsarbeit, insbesondere an Hochschulen, der Bildung einer Planspiel-AG sowie in der Organisation laufender Veranstaltungen und Kooperationen mit anderen umweltorientierten Initiativen. Mit einem Positionspapier zur ökologischen Steuerreform soll in die politische Debatte eingegriffen werden.

Zum selben Thema wird am 16. Oktober um 14 Uhr in Berlin eine öffentliche Podiumsdiskussion mit Gästen aus Politik, Wissenschaft und Wirtschaft veranstaltet. Veranstaltungsort ist das Kato im U-Bahnhof Schlesisches Tor in BerlinKreuzberg. Ein weiterer Schwerpunkt der Arbeit ist die Verbesserung der virtuellen Darstellung der VöW sowie der Kommunikation der Mitglieder über die elektronischen Medien.

Kontakt: VÖW e.V., Geschäftsstelle, Potsdamer Str. 105, 10785 Berlin, E-mail: info@voew.de, Internet: http://wwv.voew.de.

\section{- Umweltfernstudiengänge}

Die Universität des Saarlandes, die Universität Rostock und die Universität Hildesheim bieten gemeinsam die weiterbildenden Studiengänge Umweltschutz sowie Umwelt und Bildung als Fernstudienangebote an. Beide Studiengänge, wurden an der Universität Rostock entwickelt. Während der fünfsemestrige Studiengang Umweltschutz einen technischen bzw. ökologischen Schwerpunkt hat, verknüpft das Fernstudium Umwelt und Politik in drei Semestern Geistes- und Sozialwissenschaften mit einer naturwissenschaftlichen Ausbildung. Die Studiengänge richten sich an HochschulabsolventInnen, die sich neue Berufsfelder erschließen möchten oder bereits in umweltbezogenen Tätigkeiten beschäftigt sind.

Bei freien Plätzen ist die Einschreibung zu den Studiengängen für das Wintersemester 1999/ 2000 auch nach dem 15.9.99 noch möglich.

Kontakt: Fernstudienzentrum der Universität des Saarlandes, Andrea Bütterich, Tel. 0681/ 302 2063, Fax:0681/302 4666 .

Berichtigung: Folgende Grafik war in Ausgabe 3/99, S.29 fehlerhaft abgedruckt. Figure 1: Classification of companies according to the level of institutionalisation of LCA

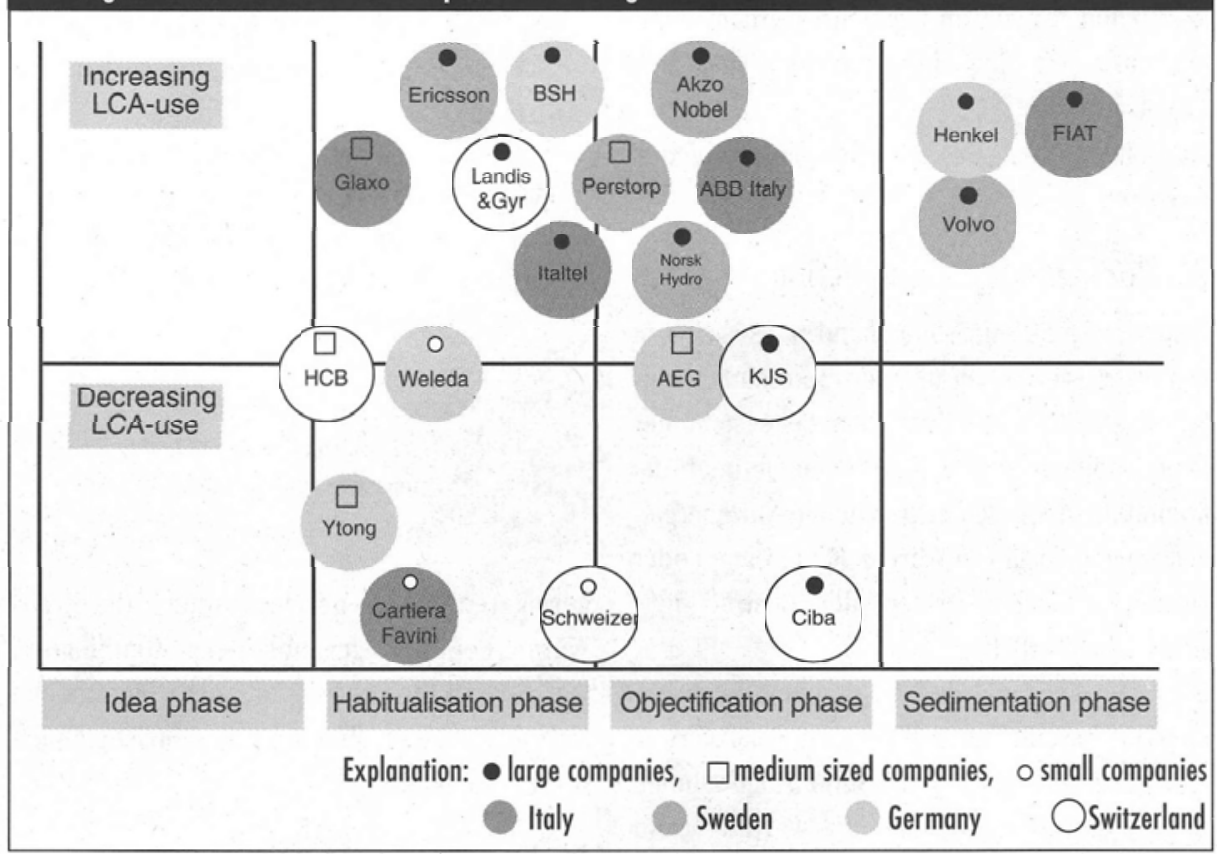

Source: adapted from Frankl, Paolo/Frieder Rubik: Life Cycle Assessment in Industry and business. Springer, Berlin et al., forthcoming

Das Finanzierungsinstrument Contracting

\title{
Rentables Energiesparen
}

Energiesparprojekte scheitern oft an einem entscheidenden Punkt: Es fehlen die nötigen Finanzmittel. Als alternative Möglichkeit, die diese Hemmnisse überwindet, gewinnt in letzter Zeit das Thema Contracting zunehmend an Bedeutung. Immer mehr Beispiele zeigen, dass diese Art des Energiesparens rentabel sein kann.

$\mathbf{W}$ Von Dietmar Graf ie kann Energie eingespart werden? Zum einen sicherlich durch eine Änderung des Verhaltens der Konsumenten. Hier besteht der Vorteil, dass keine oder nur geringe Investitionen notwendig sind. Zum anderen durch technische Verbesserungen. Dies zieht allerdings Kapitalinvestitionen nach sich, beispielsweise für neue Formen der Energieerzeugung wie Anlagen in Kraft-Wärme Kopplung oder Aufwendungen für Wärmedämmung.

Aus ökonomischer Perspektive machen Energiesparinvestitionen nur dann Sinn, wenn sie kurze Amortisationszeiten haben. Deshalb ist das wirtschaftliche Potenzial für Energiesparmaßnahmen meist wesentlich geringer als das technische. Ein Ausweg aus diesem Dilemma bietet das
Finanzierungsinstrument Contracting. Es ermöglicht, die notwendigen Anfangsinvestitionen auszulagern und die Maßnahmen durch eine Art Ratenzahlung zu finanzieren. Insbesondere innovative Projekte, die mit technischen und wirtschaftlichen Risiken verbunden sind, können so ohne Mehrkosten realisiert werden. Das Risiko trägt der Contractor.

Energiesparen bedeutet für das Energieversorgungsunternehmen (EVU) als Lieferunternehmen zunächst eine Reduzierung der Umsatzerlöse. Bietet das EVU im Gegenzug dem Kunden ein Einspar-Contracting-Projekt an, so steigert dies zwar nicht die Erlöse aus dem Strom- oder Gasverkauf, erschließt aber ein neues Geschäftsfeld, in dem wieder Gewinne gemacht werden können. 
Da Contracting die Umsetzung von Maßnahmen ermöglicht, die sonst aufgrund finanzieller Restriktionen nicht durchgeführt werden könnten, entstehen möglicherweise auch zusätzlich Arbeitsplätze.

Es können zwei Grundformen unterschieden werden:

\section{Das Anlagen-Contracting}

Beim Anlagen-Contracting handelt es sich um das vertragstechnisch und vertragsrechtlich einfachste Modell. Auf der einen Seite steht der Energieabnehmer, das Unternehmen oder die Kommune, der eine entsprechende Nutzenergielieferung in Form von Wärme, Kälte, Dampf oder Strom von einem Dritten, der Contracting Gesellschaft, erhält.

Die Dienstleistung des Contracting-Unternehmens umfasst im Regelfall Planung, Finanzierung und Realisierung der Investitionsmaßnahmen sowie die Betriebsführung, Wartung und Instandhaltung der montierten Anlagen. Die Preise für die Nutzenergielieferungen sind projektbezogen kalkuliert und werden in der Regel aufgesplittet in einen Grund- und Arbeitspreis, wobei im Grundpreis die Kapitalkosten sowie die verbrauchsunabhängigen Kosten enthalten sind und im Arbeitspreis die verbrauchsgebundenen Kosten. Alternativ kann die Kalkulation auch nach einem linearen Tarif erfolgen.

Der Contractor kann seinen Gewinn unter sonst gleichbleibenden Bedingungen nur durch Reduzierung der Kosten des Endenergiebezugs vergrößern. Es besteht der Anreiz, einzelwirtschaftlich effiziente Investitionen zur Reduzierung der Verluste bei der Umwandlung von Endenergie in Nutzenergie durchzuführen. Das ContractingUnternehmen wird also möglichst energieeffiziente Maschinen einsetzen.

Maßnahmen zur Verringerung des Nutzenergiebedarfes obliegen dem Verbraucher. Da die Vergütung des Contractors an die gelieferte Nutzenergiemenge gekoppelt ist, existiert für den Verbraucher der Anreiz, einzelwirtschaftlich effiziente Maßnahmen zur Verringerung des Nutzwärmebedarfs umzusetzen.

Ein Beispiel: Fünf Handwerksbetriebe und die Gemeindeverwaltung des münsterländischen Ortes Hopsten gehen bei der Energieversorgung des Neubaugebietes „Börnkamp“ neue Wege. Über eine gemeinsame Betreibergesellschaft versorgen sie das 12,6 Hektar große Wohngebiet mit Wärme und Strom aus einem Blockheizkraftwerk. Außer einem erheblich
Contracting Congress 11.-12.10.99

Die Niedersächsische Energieagentur veranstaltet am 11.und 12.0ktober 1999 im Kongresszentrum von Hannover den "1. Norddeutschen Contracting Kongress".

Referenten aus der Proxis informieren über die vielfältigen Möglichkeiten im Bereich Contracting. In Workshops können Erfahrungen und Anregungen gemeinsam mit den Referenten und anderen Teitnehmern diskutiert werden.

Kontakt: Niedersächsische Energieagentur GmbH, Rühmkorffstr. 1, 30163 Honnover,

Tel. 0511/96529-0, Fox 0511/96529-99, e-mail: pco-herrenhaeuser@ping-net.de

geringeren Primärenergieverbrauch der KraftWärme-Kopplung gegenüber der Kombination von reinem Strombezug aus dem öffentlichen Netz konnte der Betreiber mittelfristig einen Gewinn erzielen.

\section{- Einspar-Contracting}

Im Gegensatz zum Anlagen-Contracting sind beim Einspar-Contracting die eingesparten Energiekosten Grundlage für die Finanzierung der Maßnahmen und Investitionen des Contracting-Unternehmers. Bevorzugte Anwendungsbereiche sind Technologien, die einen hohen Energieverbrauch vermindern. Hierzu zählen vor allem Maßnahmen aus den Bereichen Beleuchtung, Klima/Lüftung, Heizung, Pumpen, Druckluft, Kälte und elektrische Antriebe.

Beim Einspar-Contracting plant, finanziert und realisiert der Contractor eine einzelwirtschaftlich effiziente Maßnahme oder ein Maßnahmenpaket zur Reduzierung der Energiekosten des Auftraggebers. Üblicherweise geht mit der Verringerung der Kosten eine Verbrauchsreduzierung einher. Die freigesetzten Gelder ermöglichen es, im Rahmen einer festgelegten Vertragslaufzeit die Investition zu refinanzieren und die übrigen vom Contractor zu erbringenden Leistungen wie Betriebsführung, Wartung und/oder Instandhaltung abzugelten.

Die Refinanzierung der erforderlichen Investitionen sowie die Vergütung für die übrigen Leistungen des Contractors werden über die Beteiligung an den eingesparten Kosten bestritten. Contractor und Auftragnehmer partizipieren an den erreichten Einsparungen, so dass für beide der Anreiz besteht, das vorhandene Kostensenkungspotential in möglichst großem Umfang zu moblisieren.
Im Idealfall kann also mittels Einspar-Contracting das gesamte Potential der einzelwirtschaftlich effizienten Maßnahmen zur Reduzierung des Energiebedarfes realisiert werden.

Beispielsweise wird die Stadt Göttingen gemeinsam mit der Niedersächsischen Energie-Agentur im Neuen Rathaus ihre erste Beleuchtungssanierung mit einem Einspar-Contracting realisieren. Ein entsprechender Vertrag wurde jetzt nach einer zweijährigen Vorbereitungszeit unterzeichnet.

Für das Rathaus hat die Niedersächsische Energieagentur mit einer Grobanalyse Energiesparpotentiale insbesondere bei der Beleuchtung nachgewiesen. Die Energieagentur wurde daraufhin mit der weitergehenden Planung und anschließenden Umsetzung beauftragt. Mit der jetzt entwickelten Lösung kann der Strombedarf mehr als halbiert werden. Dies bedeutet für das Neue Rathaus zukünftig eine Energieeinsparung von 220.000 Kilowattstunden Strom pro Jahr. Die Investitionen für die Beleuchtung werden vollständig von den eingesparten Stromkosten abgedeckt.

Contracting-Modelle schießen mittlerweile überall in Deutschland wie Pilze aus dem Boden. Außer im Energiebereich ist auch in den Bereichen Wasser und Abfall eine deutliche Zunahme an Projekten zu verzeichnen.

\section{Literafur:}

- von Braunmühl, Wilhelm: Handbuch Contracting, Krammer Verlag, Düsseldorf 1997

- Arbeitsgemeinschaft kommunaler Versorgungsunternehmen im VKU-ASEW, Köln 1996: Leiffaden Einsparcontracting - Zentrolverband des Deutschen Baugewerbes ZDB: Leiffaden Energie-Contracting

- Vereinigung Deutscher Elektrizitötswerke e.V. - VDEW, Frankfurt/Main: Contracting - Fallbeispielsammlung, Verlags- und Wirtschaftsgesellschaft der Elektrizitätswerke mbH - VWEW, 1997

- Guido Knott: Energie-Contracting. Energiewirtschaft und Technik Verlagsgesellschaft mbH, Essen, 1997

- Vereinigung Deutscher Elektriziẗtswerke e.V. - VDEW: Contracting - Wirtschaftlichkeitsbeurteilung, Verlags- und Wirtschaftsgesellschaft der Elektrizitätswerke mbH VWEW, Frankfurt am Main, 1997.

Der Autor
Dietmar Graf arbeitet seit 1998 als Redakteur
bei der ökom GmbH
Kontakt: ökom GmbH, Waltherstr. 29, 80337 Mün-
chen, Tel 089/544184-60, Fax 089/544184-99,
E-mail: redaktion@oekom.de


(c) 20I0 Authors; licensee IÖW and oekom verlag. This is an article distributed under the terms of the Creative Commons Attribution Non-Commercial No Derivates License (http://creativecommons.org/licenses/by-nc-nd/3.o/), which permits unrestricted use, distribution, and reproduction in any medium, provided the original work is properly cited. 